\title{
Integrated 3D Body Scanner with Real-Time Compensation of Breathing Movement Artifacts
}

\author{
Paolo PATETE*, Marco RIBOLDI, Guido BARONI \\ Bioengineering Department, Politecnico di Milano, Milan, Italy
}

\begin{abstract}
In the present work we introduce a new integrated body surface scanning system, especially thought for breast plastic and reconstructive surgery, made up by a programmable laser projector and a hand-held laser scanner, capable of acquiring and compensating patient breathing and involuntary movements through an optical tracking system, which is also needed to spatially localize the hand-held scanner. The programmable laser static projector is used to obtain a global but rough acquisition of patient surface, then the scanning is completed using the hand-held scanner through the scan of all hidden areas or filling the acquisition where is needed a higher detail description. The patient is fitted with a configuration of passive markers, which is used to detect patient movements at the same time of the acquisition of the laser spot projected by the "fixed" laser scanner or by the hand-held one. The use of an optical tracking system allows to obtain a real on-line compensation of the patient movements, and an automatic multiple patch connection (PCT Patent PCT/EP2009/009225). Our results show a perfect junction of different surface patches and a complete breathing and involuntary movement artifacts compensation, suggesting the presented methodology and instrumentation as a future complete solution for clinical body surface acquisition.
\end{abstract}

Keywords: breast reconstructive surgery, hand-held scanner, breathing movements compensation

\section{Introduction}

In the last few years, the use of 3D laser scanners for body surface quantitative analysis has become more and more common in breast plastic and reconstructive surgery research. This is due to the clinicians' increasing need of tools for surigical planning and surgical outcome quantitative assessment, that are nowadays common in other surgical branches like neurosurgery or orthopedic surgery. On the contrary, clinical praxis is sill anchored to traditional assessment methodologies like measuring distances among body landmarks or shooting sets of uncalibrated photographs [3], and this limits the development of technological tools specifically designed for breast aesthetic and reconstructive surgery. As a matter of fact, laser scanners commonly used in plastic surgery are specifically designed for industrial use, so they're suitable for maxillofacial surgery [2], for example, in which the surface to acquire is static and underformable, but they're hardly usable in breast surgery for three main reasons:

- If patient's surface is acquired in a single shot, using for example structured light instrumentation, in most cases it's impossible to acquire the whole surface. The acquisition of thorax lateral surface or hidden areas like the inframammary fold requires the repositioning of the patient and/or of the scanner itself $[1,3,4]$. This maneuver may lead to the deformation of breast surface due to an involuntary movement of the patient or to a different point of the breathing cycle in which the second acquisition is made and, for sure, requires an off-line registration of the surface patches, eventually introducing further errors.

- A second way to acquire patient surface is to use static laser plane projectors. The problems in this case are the same of the aforementioned methodology, but the non-istantaneous acquisition introduces also a breathing artifact present along the whole acuisition.

- Hand-held laser scanners are for sure an easy way to overcome the problem of different surface patch fusion (the acquisition is made during a single session), but the lengthened scanning duration leads to a huge deformation superimposed to the acquired surface due to the breathing movement of the patient.

In this scenario, the research we've been carrying on for the last years (covered by a international patent [6]) is focused on the developing of an advanced tool and methodology designed specifically for breast plastic and reconstructive surgery that is able to overcome all the problems just described and allows a real-time breathing artifact correction and a surface patch fusion with no intervention by the operator (apart from the scanning procedure itself). 


\section{Methods and instrumentation}

As described in the subsequent paragraphs our system is made up by a programmable laser pattern static projector, a hand-held laser scanner and an optical tracking system. To compensate breathing movements during surface acquisition, a configuration of 6 markers is fit on the patient thorax and data are analyzed through a robust mapping methodology [5] that will be described later on.

\subsection{Static laser pattern projector}

The scanner used to acquire an almost complete surface of the patient thorax (excluding all lateral areas and hidden surfaces) is an LD2000® laser pattern projector (Pangolin Laser Systems, Inc., Orlando, FL, USA). The surface acquisition is obtained through a "row-wise" scanning of the patient thorax, as shown in figure 1, and the scanning may be repeated as many times as desired in order to acquire a more dense point cloud. The projected laser spot position in the absolute coordinate system (ACS) of the laboratory is registered using an optical tracking system (see part 2.3). The LD2000® system used is capable of a nominal point projection frequency of 30000 points/s, but the acquisition duration is limited by the optical tracking system acquisition frequency.

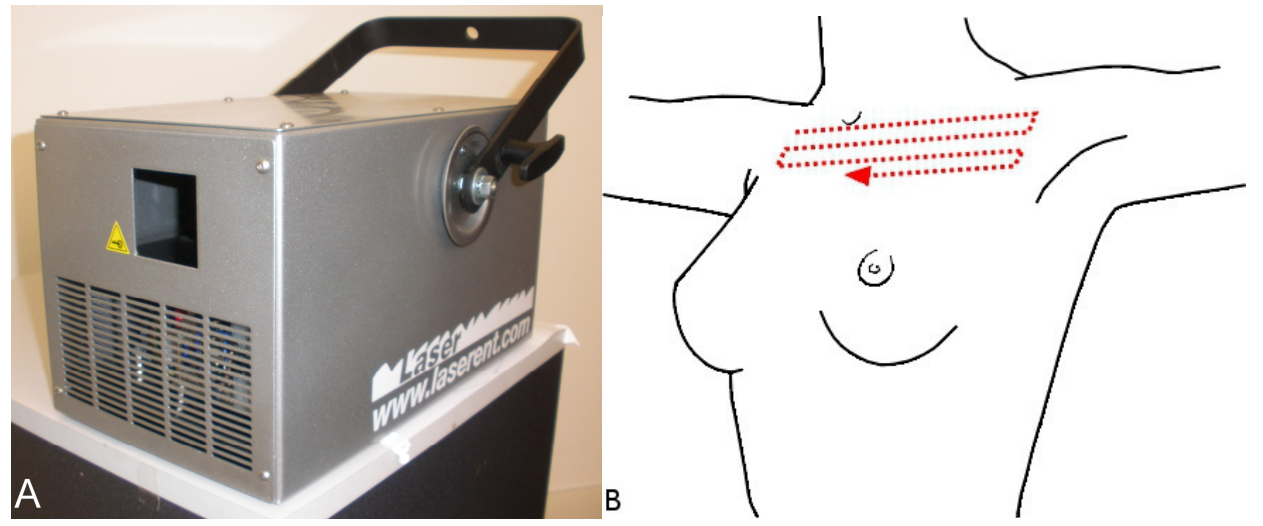

Fig. 1. Panel A: LD2000® scanning system; Panel B: Scanning pattern used for laser spot projector.

\subsection{Hand-held laser scanner}

The hand-held laser scanner consists of a spot laser source $(680 \mathrm{~nm})$ and a motion analyzer TV camera (TVC) (SMART-D, BTS S.p.A., Milan, Italy), rigidly mounted on an PMMA (polymethilmetacrylate) frame (see figure 2). To track the position of the scanner in the ARF, 5 passive markers are placed onto the frame, and their position is detected through a couple of optoelectorinc TVCs similar to the hand-held scanner one. The same passive markers are used to define a scanner coordinate system (SCS) needed to univocally determine the laser line position with respect to the mobile scanner. The calculation of the laser spot position in the ACS consequently follows a double step procedure:

1. The position on the laser line of the projected point is calculated starting from its position on the TVC sensor (requires a calibration procedure). Then, knowing the line direction in the SCS, the laser spot position with respect to the scanner is calculated.

2. Once the point is localized in the SCS, its position in the ACS is directly calculated through a mapping operation, knowing the scanner position in the ACS by tracking the 5 passive markers configuration on the PMMA frame.

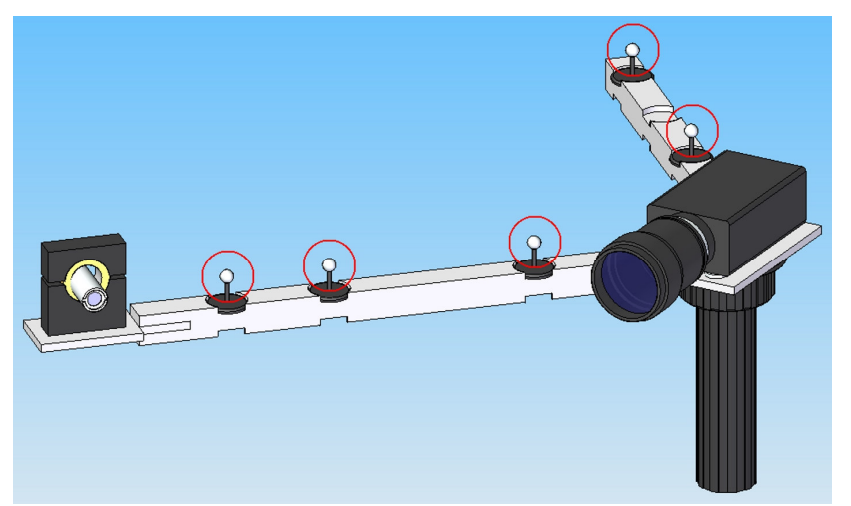

Fig. 2. The hand-held scanner: in the circles are evidenced the passive markers needed to calculate the SCS position in the ACS. 


\subsection{Optical tracking system}

The tracking system used to localize all the passive markers present in the scene and the laser spot projected on the patient surface is a SMART-D® system (BTS S.p.A., Milan, Italy), made up by three optoelectronic TVCs and a data acquisition unit (see figure 3). One TVC is used for the hand-held scanner, while the other two are actually used to localize the scanner and the laser spot. The technical characteristics of the SMART-D system are:

- Frequency acquisition: $70 \mathrm{~Hz}$

- Localization accuracy: $<0.2 \mathrm{~mm}$ (acquisition volume $3 \times 2 \times 2 \mathrm{~m}$ )

- CCD resolution: 1.4Mpix

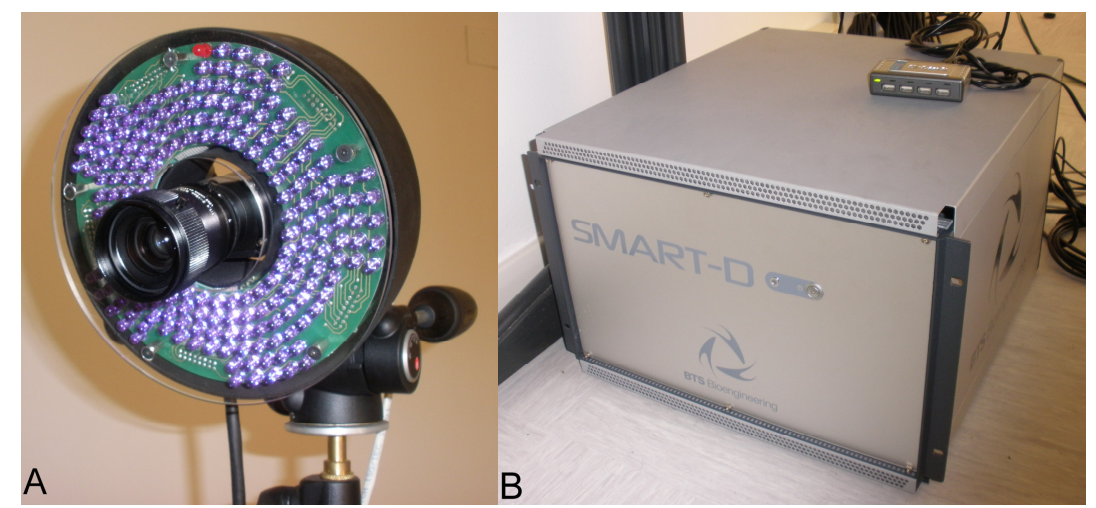

Fig. 3. The optical tracking system used. Panel A: one of the three optoelectronic TVCs; Panel B: the data acquisition unit.

\subsection{Active breathing artifact compensation}

To acquire and compensate breathing movements of the patient, a configuration of 6 passive markers is fitted on patient thorax surface. They're positioned on the following body landmarks (see figure 4):

1. jugular lacuna $(n \circ 1)$

2. bilaterally, on the clavicle, at half-distance between the jugular lacuna and the acromion $\left(n^{\circ} 2-3\right)$

3. xiphoid process $\left(n^{\circ} 4\right)$

4. medial edge of the rib cage, few centimeters above the umbilical line (these latter are the same considered for the breathing acquisition used in the respiratory gating method; $n^{\circ} 5-6$ )

To correct in real time the acquired point position in order to compensate breathing movement and to connect different surface patches, a further procedure has to be followed. First the point is mapped in the patient coordinate system (PCS) defined by the six passive markers, then the transformation matrix that describes the PCS position in the current frame with respect to its position in a reference frame (e.g. the first) is calculated. By multiplying this transformation matrix with the laser spot coordinates in the PCS it is possible to describe the patient surface in a unique coordinate system, without any artifact due to the breathing movement. Moreover, localizing each point acquired in a single coordinate system, the fusion of different surface patches is automatically achieved, with no further intervention requested to the operator.

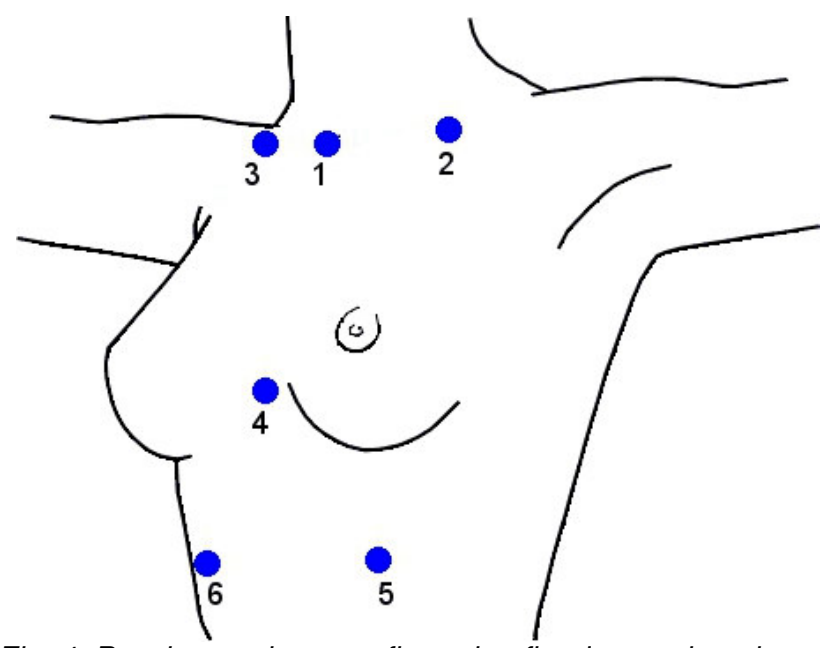

Fig. 4. Passive markers configuration fitted on patient thorax. 


\subsection{Acquisition tests}

To evaluate the accuracy of the acquisition system in a controlled setup, an anthropomorphic phantom was acquired 14 times, and the resulting surfaces were compared with a CT scan of the phantom. The RMSE of the distances between the points acquired and the CT surface was then calculated in order to quantify the global accuracy of the acquisition system, not considering the errors that the active breathing algorithm may introduce.

\subsection{Patient acquisition protocol}

Patient surface scanning session follows a specific protocol. Patients are positioned on a chair with both arms stretched horizontally and the PCS passive markers are placed on the patient thorax. The acquisition is split into two sessions: a first scan is made with the LD2000® system, then the surface is completed by the operator with the hand-held scanner. Each phase is checked through The operator runs an ad hoc software designed to give a real-time feedback through a 3D representation of the acquired point cloud (see figure 5). The only off-line procedure requested is a surface filtering in order to correct any mislocalized point. The evaluation of the accuracy of the breathing compensation technique is made through a qualitative evaluation of morphological similarity of the surface meshes with respect to the subjects' clinical appearance (assessed by means of a rich photographic dataset) lacking of any surface reference data obtained from volumetric imaging (such as CT or MRI), which would have been in any case affected by motion artifacts and provide the morphology of a laying and not of a sitting patient.

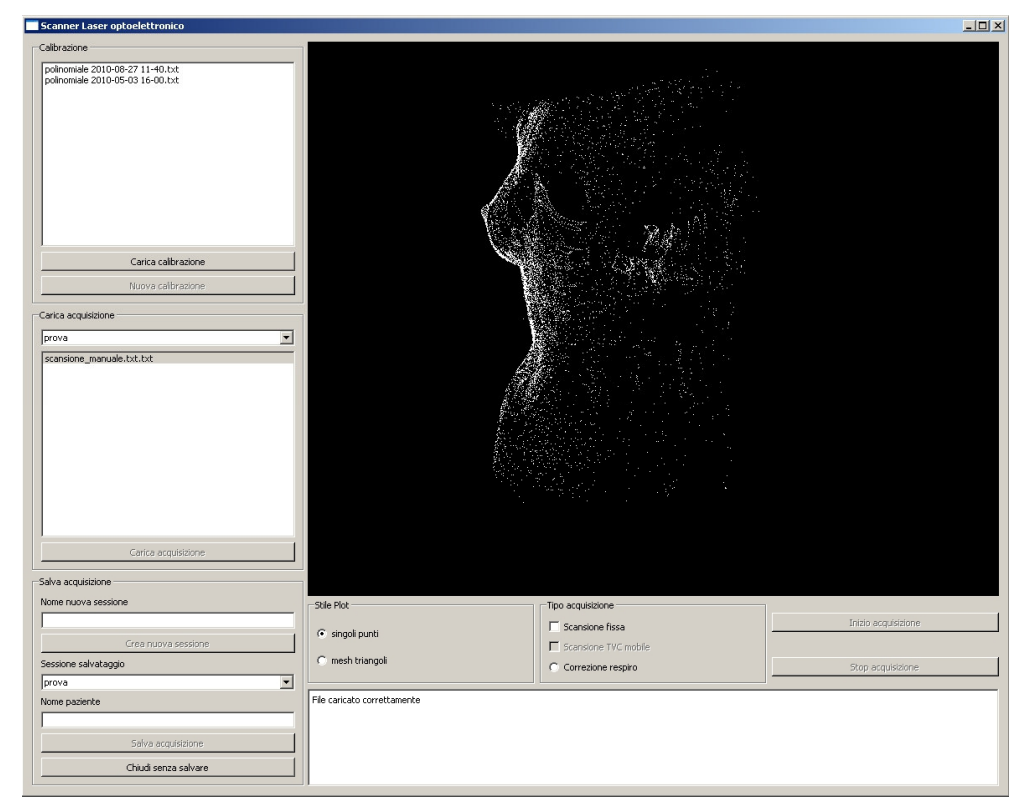

Fig. 5. User interface of the acquisition and control software. A real-time feedback is given to the operator while the scanning is being executed.

\section{Results}

\subsection{Scanner accuracy tests}

The results concerning the 14 anthropomorphic phantom surfaces, made up by $3480.9 \pm 477.0$ points (mean \pm std. deviation, $O \pm \bullet$ ), showed a systematic displacement error of $0.227 \pm 0.284 \mathrm{~mm}(O \pm \bullet$ ) on the $X$ axis, $0.216 \pm 0.211 \mathrm{~mm}(\mathrm{O} \pm \bullet$ ) on the $Y$ axis, $0.280 \pm 0.162 \mathrm{~mm}(\mathrm{O} \pm \bullet)$ on the $Z$ axis (almost coincident with the bisector of the angle formed by the optical axes of the static TVCs). The RMSE of the point to surface distance was $1.732 \pm 0.736 \mathrm{~mm}(\mathrm{O} \pm \bullet)$.

\subsection{Patient acquisitions}

Figure 6 shows an example of the results concerning a patients' acquisitions. is shown in figure 4. The top right panel of the figure shows the mesh created starting from the points acquired without any breathing movement compensation algorithm: the presence of the breathing artifact is evident. The upper right panel shows the mesh created from the corrected points, without any filtering process applied to the mesh. The lower panels show respectively the filtered mesh and a picture of the patient. The same four panels are shown in figure 7. 


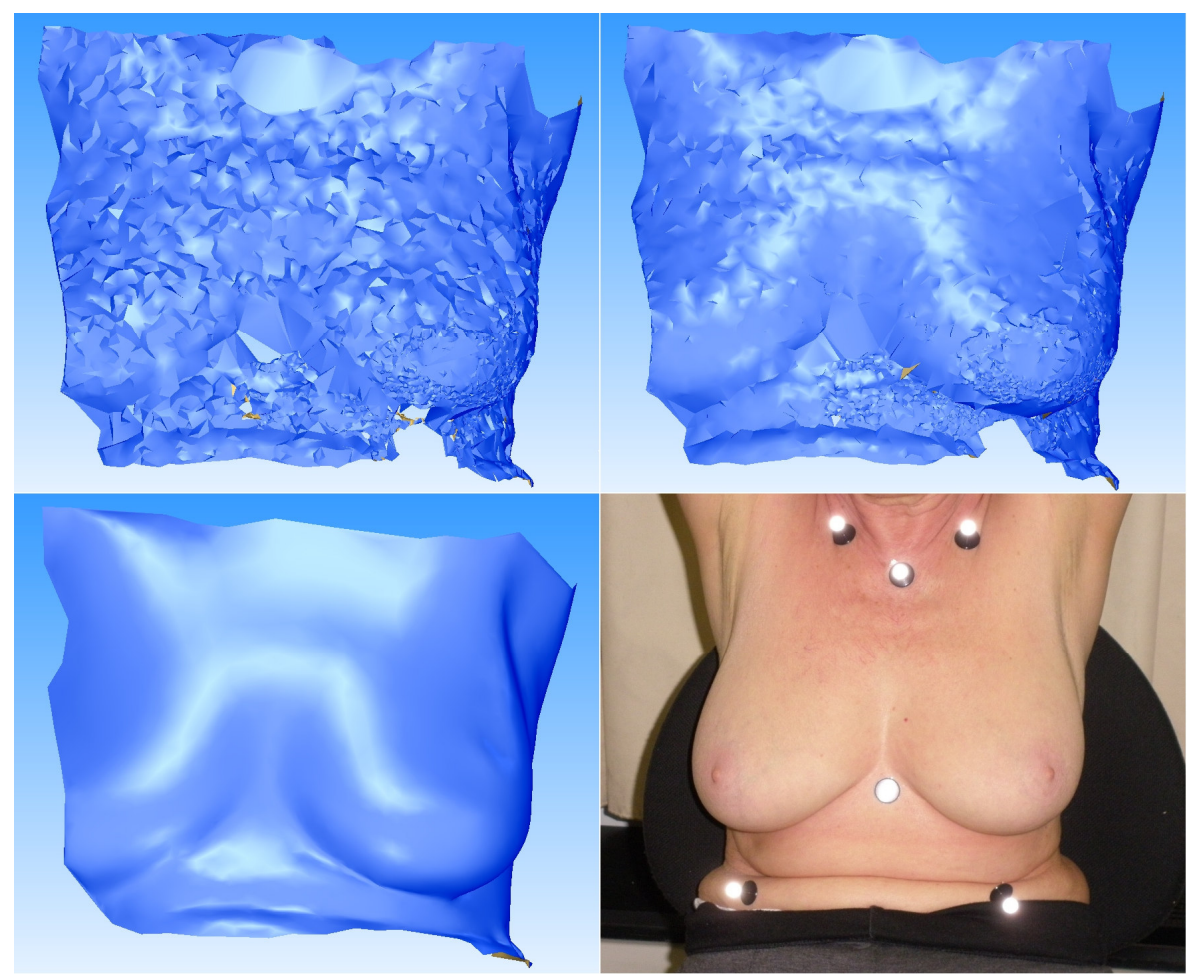

Fig. 6. Patient acquisition results: front. See text for details

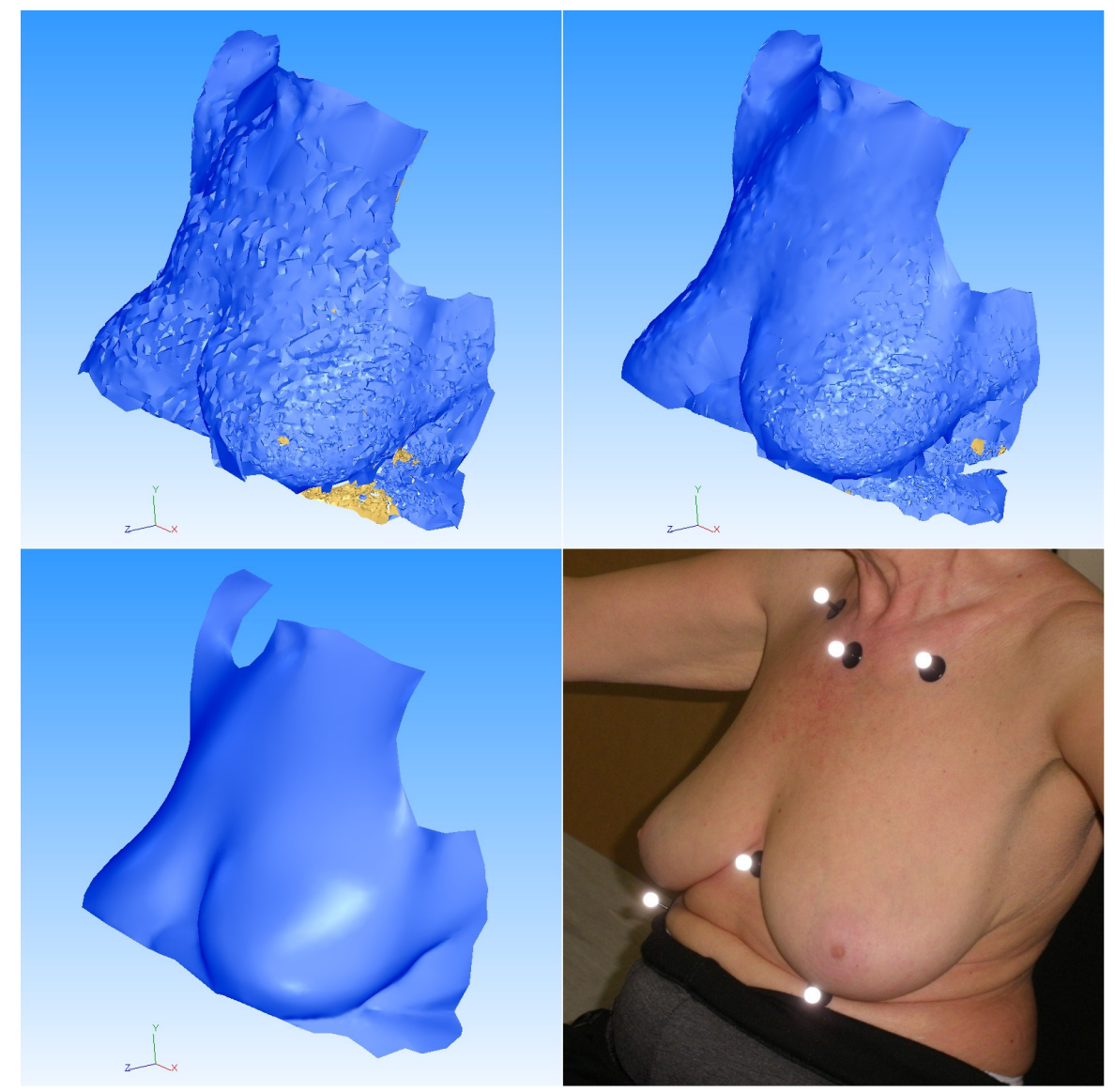

Fig. 7. Patient acquisition results: side. See text for details 


\section{Conclusions and discussion}

In body surface acquisition, the main problem is represented by patient's breathing movements. Industrial scanners, generally used for subjects surface acquisition, don't allow an on-line acquisition and compensation of such movements, leading to noisy surfaces that need a post-acquisition process to reduce movement artifacts. Moreover, many scanning sessions are required to completely acquire patients surface, possibly introducing errors in the patch fusion process. The integrated instrumentation/methodology proposed shows a very good performance either as common scanning system, due to its high accuracy surface reconstruction, or as integrated system capable of acquiring and compensating patient's breathing movements in real-time. Another remarkable feature is the automatic multiple patch registration: no off-line procedure is requested to create a complete model of patient surface. These characteristics indicate the presented scanning system as a valuable tool for clinical patient scanning, above all in breast plastic and reconstructive surgery, due to the little training required to the operators and the short time needed for a complete scan of the patient (only a couple of minutes).

\section{References}

1. Catanuto, G., Spano, A., Pennati, A., Riggio, E., Farinella, G. M., Impoco, G., Spoto, S., Gallo, G., Nava M. B., (2008): "Experimental methodology for digital breast shape analysis and objective surgical outcome evaluation", J. Plast. Reconstr. Aesthet. Surg. Vol. 61, pp. 314-318.

2. Harrison, J. A., Nixon, M. A., Fright, W. R, Snape, L. (2004): "Use of hand-held laser scanning in the assessment of facial swelling: a preliminary study", Br. J. Oral. Maxillofac. Surg. Vol. 42, pp.8-17.

3. Kovacs, L., Yassouridis, A., Zimmermann, A., Brockmann, G., Wöhnl, A., Blaschke, M., Eder, M., Schwenzer-Zimmerer, K., Rosenberg, R., Papadopulos, N. A., Biemer, E., (2006): "Optimization of 3-dimensional imaging of the breast region with 3-dimensional laser scanners", Ann. Plast. Surg. Vol. 56, pp. 229-236.

4. Losken, A., Fishman, I., Denson, D. D., Moyer, H. R., Carlson G. W., (2005): "An objective evaluation of breast symmetry and shape differences using 3-dimensional images", Ann. Plast. Surg. Vol. 55, pp. 571-575.

5. Patete, P., Riboldi, M., Spadea, M. F., Catanuto, G., Spano, A., Nava, M., Baroni, G., (2009): "Motion compensation in hand-held laser scanning for surface modeling in plastic and reconstructive surgery", Annals of Biomedical Engineering Vol. 37, pp. 1877-1885.

6. Baroni, G., Riboldi, M., Patete, P. (2009): "System And Method For Advanced Scanning And For Deformation Simulation Of Surfaces”, PCT Patent PCT/EP2009/009225. 